\title{
LETTERS
}

alternative. While 15\% of GDPs referred their patients with TMJ problems immediately, 40\% referred their patients after 3-6 months, presumably after a period of conservative management which is in line with that which most TMJ surgeons feel is appropriate. The majority of dentists were unclear about the surgical interventions for TMJ dysfunction with nearly half unaware that TMJ replacement existed. Over 90\% of GDPs thought that they would benefit from a refresher course.

Patients with TMJ problems, often presenting with varied complaints, are encountered by dental practitioners on a daily basis. It is important to have a sound knowledge and understanding to formulate a treatment plan or to refer when appropriate. These results highlight that TMJ examination, knowledge and pathology is not well understood and they suggest that more training in TMJ pathology at an undergraduate level and during vocational training is warranted.

K. Kassam

Senior Oral \&t Maxillofacial Specialist Trainee

1. Al-Ani M, Gray R M J, Davies S J, Sloan P, Worthington $\mathrm{H} \mathrm{V}$. Anterior repositioning splint for temporomandibular joint disc displacement (Protocol for Cochrane Review). Volume 1. The Cochrane Library, 2003.

DOI: sj.bdj.2013.1051

\section{HPV AND THROAT CANCER}

Sir, a recent news item in your columns about human papillomavirus (HPV) (BDJ 2013; 215: 270) entitled HPV linked to a third of throat cancer cases concluded 'These results could have important implications for early diagnosis and risk assessment, as well as the clinical impact of the HPV vaccine, broadening its protective sphere against not only cervical cancer, but oropharyngeal cancers and others, though further research is needed.'

Readers will be interested to learn that there is published a large study conducted on 5,840 sexually active women in Costa Rica (ages 18-25 years) comparing the effects of an HPV vaccine with placebo, in which, after four years, mouthwash samples showed only one woman after vaccine was orally HPV-infected but 15 women after placebo vaccine were infected. ${ }^{1}$

\section{Scully} By email

1. Herrero R, Quint W, Hildesheim A et al. Reduced prevalence of oral human papillomavirus (HPV) four years after Bivalent HPV Vaccination in a randomized clinical trial in Costa Rica. PLOS ONE 2013; 8: e68329.

DOI: sj.bdj.2013.1052

\section{FINE CRUSTING BLISTERS}

Sir, as junior trainees within the hospital dental system we see a concentrated number of cases referred by dental practitioners to maxillofacial departments as emergencies. The majority are usually infections of dental origin, but at times we do see the weird and wonderful or classic presentations of fairly common oral medicine conditions depicted in oral medicine textbooks. We have always found oral medicine an intriguing topic, but have noticed a number of clinicians once they have entered the realms of practice, lose the penchant for it. It is not only us in the dental profession that have trouble with unusual presentations of certain common pathology but we have noticed our medical counterparts are also guilty.

A recent case was a 76-year-old lady referred to the emergency department by her general medical practitioner complaining of pain and an unusual skin sensation over the left side of her face and eye. The case was referred to us as an acute cellulitis of dental cause as she was treated by her dental practitioner three days prior. An acute periradicular periodontitis was the assumed diagnosis as it appeared that attempts were made by the GDP to extract the lone standing 37 . The failed extraction resulted in a broken crown. Intraorally this was asymptomatic at presentation (Fig. 1). The area had marked erythema and swelling along the maxillary distribution of the

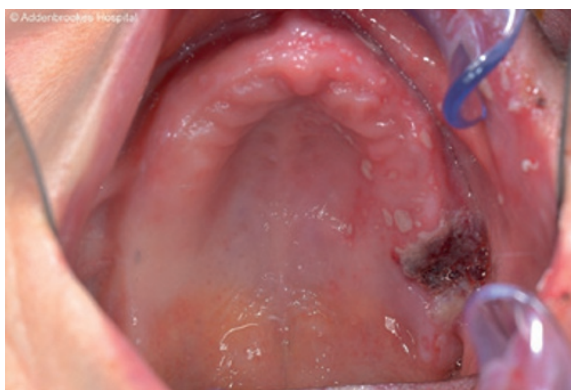

Fig. 1 Intra-oral view

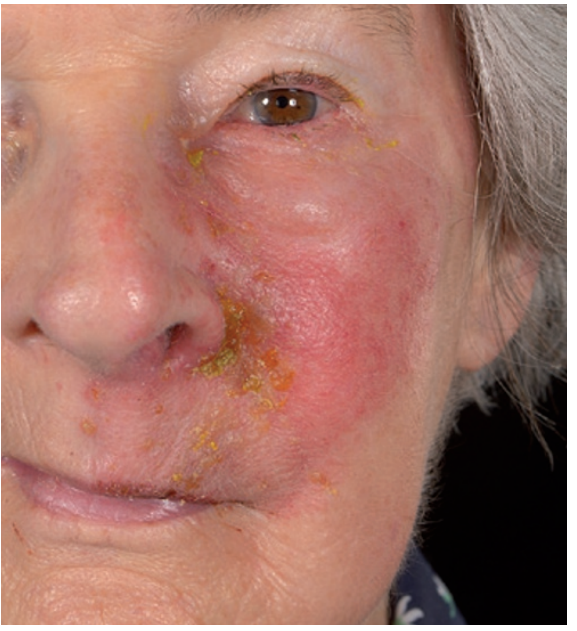

Fig. 2 Patient displayed blisters and erythema

trigeminal nerve. There was increased periorbital swelling, lacrimal excretions, and fine crusting blisters in the left infra orbital region. She was apyrexic with a CRP of 14 with a normal WBC. She was started on co-amoxiclav by the dental SHO who also did not pick up the diagnosis from her presentation and assumed the working diagnosis as a dental infection. Ward rounds the following morning revealed a textbook clinical picture of V2 shingles demarcation with the blisters and erythema pathognomonic of this disease (Fig. 2). Viral swabs were taken and PCR analysis confirmed this diagnosis. It is through seeing cases like this that the diseases become etched in our memories and remind us of the importance of oral medicine. This is why we plead to our professional colleagues to keep current with their oral medicine.

J. Philip, R. Walton, G. Bourne, M. Cameron

Cambridge DOI: sj.bdj.2013.1053 